\title{
INFLUENCE OF TRAIN CONTROL SYSTEM ON RAILWAY TraCK CAPACITY
}

\author{
HARAMINA, H.; BRABEC, D. \& GRGIC, D.
}

Abstract: The basic advantage of the train control methods based on the moving block technology compared to the fixed blocks where the headways of the slower trains, regarding their longer blocking times affects negatively the line capacity, lies in the fact that the position and the length of the moving blocks adapts to the position, dynamic characteristics, and actual speed of the successive train. Regarding a higher possibility for influence on the train movement characteristic during the moving block train operation, better traffic fluidity with more regular traffic flow can be achieved. Considering this fact an amount of regular recovery and buffer times needed for railway timetable stability can be decreased and thus more train paths in a particular dedicated time window can be added. In this way the moving block technology can increase the capacity of a railway line, especially in case of high density lines with significant heterogeneity of traffic.

Key words: train control system, railway infrastructure capacity, moving block
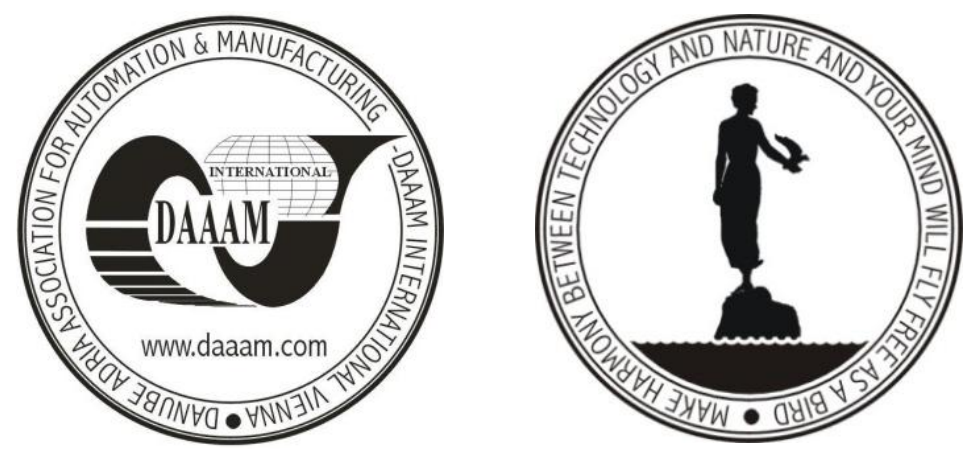

Authors' data: Dr. Sc. Haramina, H[rvoje]; dipl.ing. Brabec, D[ean]* Dr. Sc. Grgić, D[amir]**; *University of Zagreb, Faculty of Transport and Traffic Sciences, Vukelićeva 4, Zagreb, Croatia, **Hrvatske željeznice, Mihanovićeva 12, Zagreb, Croatia; hrvoje.haramina@fpz.hr, dean.brabec@fpz.hr, damir.grgic@ @znet.hr

This Publication has to be referred as: Haramina $\mathrm{H}$ [rvoje]; Brabec, $\mathrm{D}$ [ean] \& Grgić, D[amir] (2012). Influence of Train Control System on Railway Track Capacity, Chapter 36 in DAAAM International Scientific Book 2012, pp. 419-426, B. Katalinic (Ed.), Published by DAAAM International, ISBN 978-3-901509-86-5, ISSN 1726-9687, Vienna, Austria

DOI: $10.2507 /$ daaam.scibook.2012.36 
Haramina, H.; Brabec, D. \& Grgic, D.: Influence of Train Control System on Rail...

\section{Introduction}

In regular railway operation movements procedures for train separation are required that work independently from the train driver visibility range. Main reason for this is the fact that braking distances of trains, concerning their big masses and relatively low adhesion between train wheels and rails, are usually longer then sight distance of the train driver.The division of the open railway line into several blocks allows simultaneous sequence of several successive trains on the open railway line between two railway stations and results in its greater capacity (Badanjak, et al., 2007).

One of the essential problems in this type of protection is that the open railway line is divided into fixed blocks of the pre-determined length. The basic rule of rail transport safety, namely, lies in the fact that the minimal block length has to contain at least the value of the longest stipulated train braking distance on this section of the railway line, and since this distance is different for different trains the longest one is always the valid one (Pachl, 2004). Such division of the railway line often fails to be adapted to those trains that have shorter braking distances in which the headways of the slower trains on the open railway line divided into fixed blocks longer than those that, regarding their dynamic characteristics, and especially their braking distances, would be really necessary, affects negatively the line capacity and therefore represents one of the essential reasons of reduced efficiency of rail traffic. In this paper different train control methodologies will be compared and their influence on railway line capacity will be presented.

\section{Comparison of various Train Control Methods regarding Line Capacity}

Train control methods that encompass the division of open railway line into several blocks include:

- train control on open railway line divided into fixed blocks by discrete influence on the train;

- train control on open railway line divided into fixed blocks by continuous influence on the train;

- train control on open railway line by the application of the safety moving block system.

When the open railway line is divided into several fixed blocks that can be controlled regarding occupancy by railway vehicles the length of distance spacings depends on the necessary length of the blocks that maximally meet the requirements for safe and efficient operation of rail traffic. The rule is that the minimal length of the block has to contain at least the value of the longest stipulated train braking distance on this railway line section. In case of such method of train control on the open railway line, several trains can operate simultaneously in one direction, which increases the line capacity, Fig.1. 


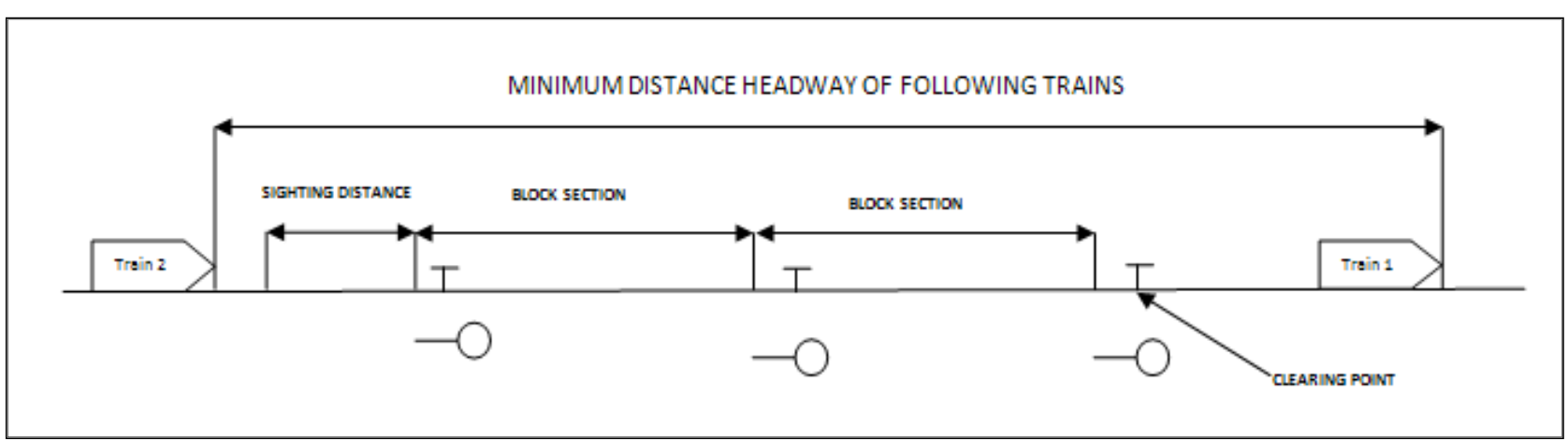

Fig.1. Separation of trains in fixed block operation

In case of such operation method the Automatic Train Protection system prevents through its automatic action the train from running along uncontrolled train route on the open railway line when the person operating the train, for any reason whatsoever does not perform the action predicted by the aspect of the block signal. In that case the train protection can be realised by installing inductive balises which act in accordance with the aspect of the block signal on the equipment installed onboard train, which allows, if necessary, train velocity control or its automatic stopping if the train running method endangers the traffic safety.

In train control system in which the system of cabin signalisation is applied by installing additional devices in the form of electronic balises and electromagnetic loops for the transmission of coded information the usage of the railway line can be additionally improved by increasing its capacity and the traffic safety can be increased by additionally influencing the method of vehicle running in specific traffic situations. An example of such a train control system is also the ETCS Level 1 (European Train Control System Level 1) in which the control information is sent in a discrete way via special track balises called Eurobalises to the train by means of radio connection. Based on the received control information the computer onboard train continuously controls and calculates the speed profile and train braking curve. One of the essential problems in this method of protection is certainly the fact that the open railway line is divided into fixed blocks of pre-defined length. This length does not correspond equally to different types of trains regarding their relevant characteristics such as acceleration, braking, maximal technical speeds, masses, lengths, etc., and this results in the reduction of the possibility of optimal rail traffic operation. Moreover, the control information that can affect the optimisation of train traffic in certain unplanned situations in train traffic can be transferred to the train only at certain points on the railway line which additionally represents a barrier in the optimisation process of the real-time train control. This is precisely the reason for the tendency to apply the train control system with continuous influence on the train.

Unlike the discrete influence on the train, namely, in case of continuous influence on the train the control information can be transferred to the train at any moment during its running along an open railway line. In this way the train can respond at any moment to the changes in traffic. Besides, the application of continuous action on the train in the process of control allows the increase in speed. Since the length of blocks is determined regarding the length of the braking distance 
of a certain train and its maximal speed, for the successive train to be able to run at a speed higher than the speed for which the braking distance of the respective train does not exceed the block length of the open railway line to which the train is arriving, it is necessary to provide a longer length of the free space in front of the successive one so as to avoid its conflict with the preceding train. This can be solved in several ways.

The first is to increase the lengths of fixed blocks with the reduction of their number, thus in case of the running of slower trains there is the problem of reduced line capacity.

Another is to organize the sequence of successive trains on three blocks which again in case of the operation of slower trains can cause reduced line capacity. It is precisely for these reasons that the need occurs to solve the mentioned problems by the application of continuous influence on the train. Some of the better known examples of solutions of control by the application of continuous influence on the trains in Europe are the systems LZB, TVM and ETCS Level 2.

The train control by the application of the LZB system (continuous influence on the train) only those trains are continuously influenced that are equipped by devices of this system, by transfer of the control information via inductive loops laid on the tracks. The train control, namely, by the application of the LZB system is most frequently performed parallel with train control using the classical system with the division of railway lines into fixed blocks and by discrete influence on the train, with high-speed trains using LZB, and all the others using the classical system of train control. The railway line for the running of trains controlled by the described system of discrete influence on the trains that operate along fixed blocks, based on the aspect of block signals, is only supplemented by the LZB system that directly acts only on those trains that are equipped for this train control method. Thus, trains controlled by the LZB system by means of cabin signalisation receive the control information about the maximally allowed running speed on a certain section of the protected route which is displayed onboard train by means of an interface to the engine driver. This is precisely the reason why the train can reach higher speed also on the railway lines where its braking distance at that speed is longer than the length of blocks, unlike the case in which, if it were controlled by the aspects of the block signals its braking distance had to be shorter or equal to the block length. Thus, in this case, the solution has been reached which increases the running speed of certain trains on the open railway line without affecting the length of the blocks that remain adapted to the running of slower trains controlled by the system that understands the traffic regulation by means of block signals. The control of the tracks condition and the integrity of trains are performed on the basis of the control of the occupancy of the tracks of each individual fixed block.

The TVM system (Transmission Voie-Machine - "transmission from railway line to train") also has a similar operation principle which is applied on the highspeed railway lines (Ligne à Grande Vitesse - LGV) in France.

Unlike LZB and TVM, in the ETCS Level 2 system the continuous transmission of control information is performed in the wireless way by means of GSM-R system 
(Global System for Mobile Communications for Railways) which originated as part of the ERTMS project started by the International Railway Union (UIC) as result of the tendency for technical harmonisation of railways in the countries of Europe, and wider.

Unlike the mentioned method of train control whose basic characteristic is the existence of the so-called fixed blocks that are pre-dimensioned and set depending on the rail traffic characteristics on a certain railway line and separated by signals, there is also the train control method in the so-called moving block. The moving block train control system does not require the track circuits or track contacts with axle counters in order to determine the position of the trains and their integrity but it rather relies on the continuous two-way digital radio communication between every controlled train and the control centre as well as on the equipment onboard train which checks its integrity.

In case of control systems that include the application of the train control method in the moving block, the railway lines are usually divided into the control areas where each is controlled by means of a computer at the train control centre with the transmission of control information by means of radio communication system, fig. 2.

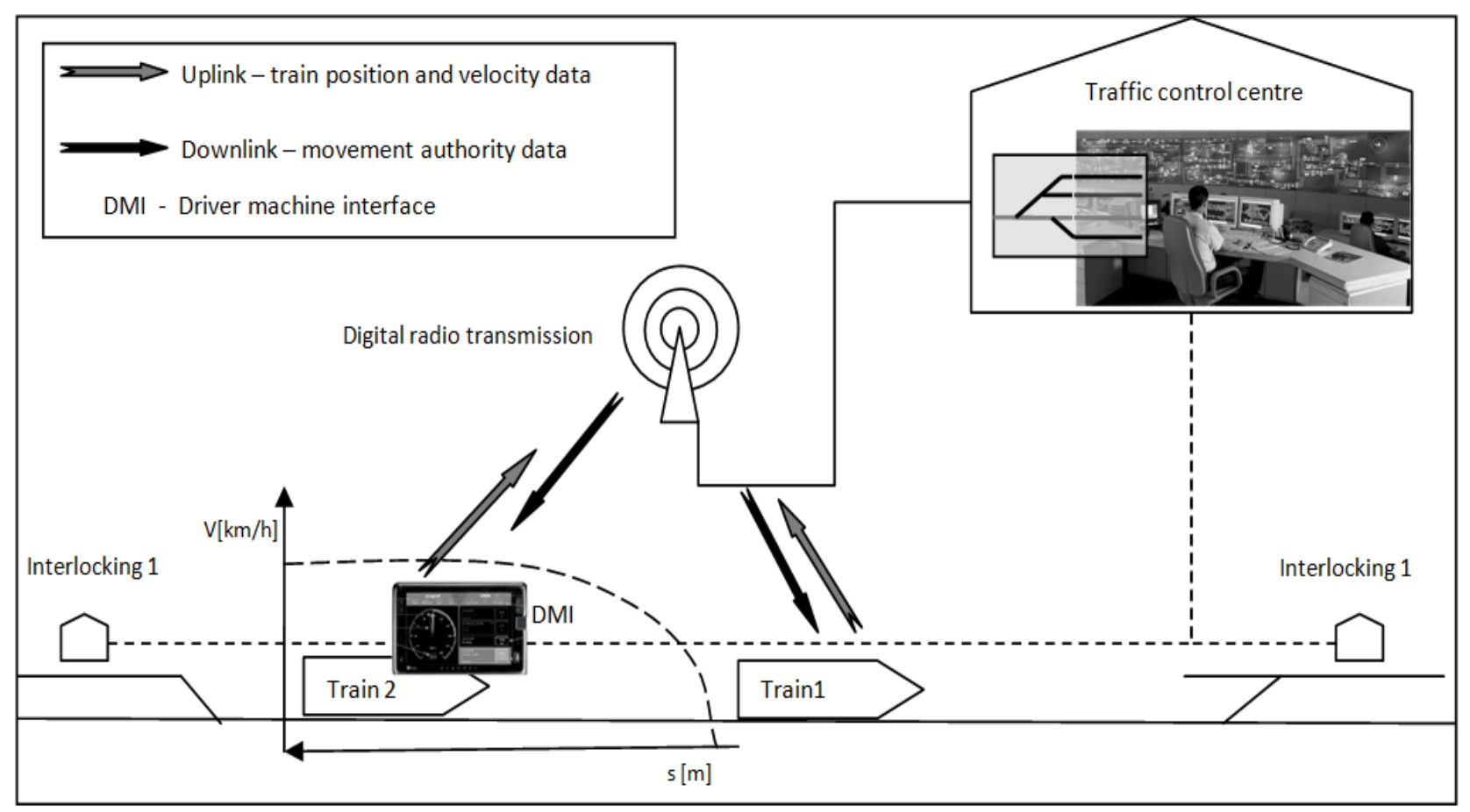

Fig. 2. Basic principles of a moving block system

Every train transmits data about its identity, position, direction of movement and speed to the computer that controls the control area in which a certain train is positioned. Based on the gathered data the computer performs the calculation of the necessary length of the gap between the trains that are within its region. The radio communication connection between each train and the computer is continuous so that the computer has all the data on the position of all the trains within its area at any moment. The computer forwards to every train the data on the position of the train it 
Haramina, H.; Brabec, D. \& Grgic, D.: Influence of Train Control System on Rail...

follows and stipulates the curve of braking necessary to stop the train before it catches up with the train ahead.

The basic advantage of the moving block system over the fixed block systems lies in the fact that the position and length of the moving blocks is adapted to the required running method to meet the planned objectives of traffic control determined on the basis of the position, dynamic characteristics and actual train speeds on a certain railway line section, Fig. 3.

\section{A.) Fixed block train separation}

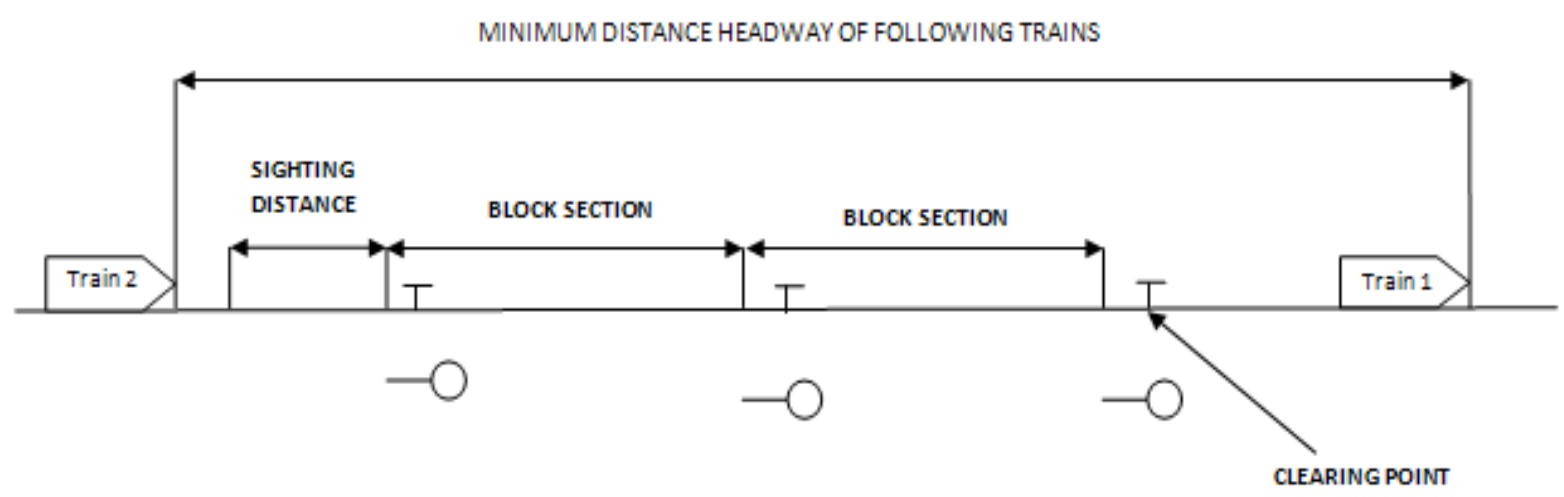

B.) Moving block train separation

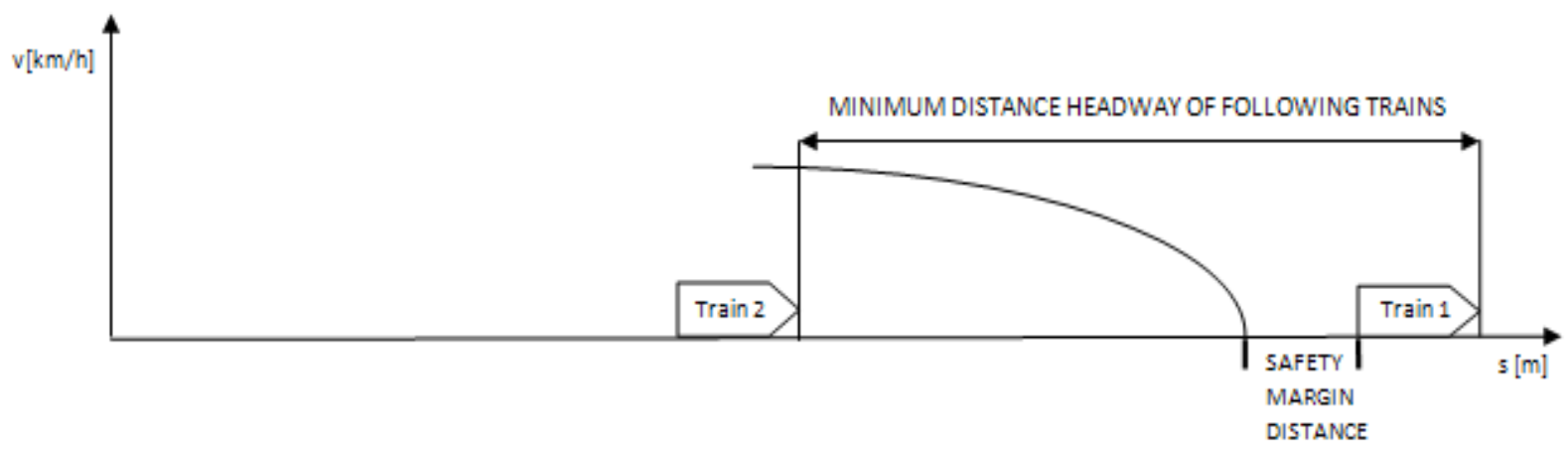

Fig. 3. Comparison of train separation distance between fixed and Moving Block principle

Thus, notable benefit by using of moving block technology is its impact on capacity of railway lines. Namely, regarding a higher possibility for influence on the train movement characteristic during the moving block train operation, better traffic fluidity with more regular traffic flow can be achieved (Hansen \& Pachl, 2008). 
Considering this fact an amount of regular recovery and buffer times needed for railway timetable stability can be decreased and thus more train paths in a particular dedicated time window can be added. In this way the moving block technology can increase the capacity of a railway line, especially in case of high density lines with significant heterogeneity of traffic.

Regarding a higher possibility for influence on the train movement characteristic during the moving block train operation, better traffic fluidity with more regular traffic flow can be achieved. Considering this fact an amount of regular recovery and buffer times needed for railway timetable stability can be decreased and thus more train paths in a particular dedicated time window can be added (Rudolph, 2003; Hansen, 2006). In this way the moving block technology can increase the capacity of a railway line, especially in case of high density lines with significant heterogeneity of traffic.

Another very important benefit of using moving block technology is lineside equipment cost reduction. Namely, lineside train detection equipment has direct impact on railway system efficiency.

Crucial part of such equipment is the signalling equipment and infrastructure (Toš, 2011). Costs for the signalling infrastructure represent a significant part of overall railway infrastructure life cycle costs. Namely, this part of railway infrastructure requires a large amount of expensive line side hardware equipment which is exposed to variable climatic conditions, wear, vandalism, theft and heavy usage. This results in high maintenance requirements regarding labour force and spare parts.

By usage of moving block technology signalling infrastructure costs can be reduced thanks to following cost saving factors:

- Removing train detection and integrity equipment from track to train;

- Minimizing signal cables by controlling elements via radio communication;

- Minimizing maintenance costs of track side equipment.

\section{Conclusion}

The basic advantage of the moving block system over the fixed block systems lies in the fact that the position and length of the moving blocks is adapted to the required running method to meet the planned objectives of traffic control (Haramina et all., 2011). Namely, the train control systems that are based on the so-called fixed blocks are based on the rule that only one train is allowed on one block.

This running method has several marked drawbacks that cause the lack of flexibility of railway traffic. In this case the length of the block is set for all the types of railway vehicles regardless of their different running speeds and braking characteristics. Thus large safety distances of the fixed block as required by fast trains with longer braking distances are unnecessarily imposed also for the slower trains since the minimal length of the fixed block, among other things, is determined by the needs of the longest minimally necessary braking distance of trains that can operate on a certain railway line. 
Haramina, H.; Brabec, D. \& Grgic, D.: Influence of Train Control System on Rail...

Unlike the train control by methods based on the fixed block, the method based on the moving block does not require track circuits or track contacts with axle counters in order to determine the position of trains and their integrity but rather relies on continuous two-way digital radio communication between every controlled train and the control centre and the equipment onboard train which checks the train integrity.

The basic advantage of the train control methods based on the moving blocks compared to the fixed blocks lies in the fact that the position and the length of the moving blocks adapts to the position, dynamic characteristics, and actual speed of the train in the area. This fact can bring a whole number of advantages regarding the realisation of the planned timetable, especially on the sections of the rail network with extremely busy traffic.

The further research will include the new computer model for simulation of railway traffic based on the moving block technology. By its usage it will be possible to determine the influence of moving block technology on timetable reliability and energy consumption of trains.

\section{References}

Badanjak, D.; Bogović, B.; Jenić, V. (2007). Organizacija željezničkog prometa, Organizing of Railway Transport, University of Zagreb, Faculty of Transport and Traffic Siences, ISBN 953-243-012-1, Zagreb

Hansen, I. A.; Pachl, J. (2008). Railway Timetable \& Traffic, Eurailpress, ISBN 9783-7771-0371-6, Hamburg

Giannettoni, M.; Savio, S. (2002). Traffic management in moving block railway systems: the results of the EU project COMBINE. Computers in Railways VIII, Ed. J. Allen, R.J. Hill, C.A. Brebbia, G. Sciutto and S. Sone, pp. 953962. WIT Press, ISBN: 978-1-85312-913-1, Southampton

Toš, Z. (2011) http://www.fpz.hr - Automatizacija u željezničkom prometu, Railway Automation, University of Zagreb, Faculty of Transport and Traffic Sciences, Accessed on: 2012-06-18

Hansen, I. A. (2006). State-of-the-art of railway operations research, Computers in Railways X, WitPress, pp. 565- 577

Haramina. H.; Brabec, D.; Stefančić, I. (2011). Influence of Train Control System

Characteristic on Railway Infrastructure Capacity, Proceedings of 22nd

DAAAM World Symposium "Intelligent Manufacturing \& Automation: Power of Knowledge and Creativity"Katalinic, B.(Ed)., pp. 0357-0358, ISBN 978-3901509-83-4,23-26th November 2011, Vienna, Austria

Pachl, J. (2004). Railway Operation and Control, VTD Rail Publishing, ISBN 09719915-1-0, Mountlake Terrace

Rudolph, R. (2003). Allowances and margins in railway scheduling. In: Proceedings of WCRR, Edinburgh. pp. 230-238 